\title{
Recursos on-line sobre experiencias innovadoras en competencias transversales para profesores y alumnos
}

\author{
Salvador Calvet Sanz, Héctor Moreno Ramón, Sara Ibáñez Asensio, , Ana Isabel \\ Jiménez Belenguer, Ignacio Guillén Guillamón, Marta Cabedo Fabrés, Alfonso \\ Martínez García, Miguel Ferrando Bataller \\ Universitat Politécnica de Valencia, Valencia, Spain (salcalsa@upvnet.upv.es)
}

\begin{abstract}
One of the most relevant threats that teachers face when developing core skills in higher education is how to do it in the real situation of their classes. This may result in demotivation of teachers to achieve this challenge. At this moment, an important training task is necessary for both teachers and students, particularly regarding how to develop and evaluate these skills. The general objective of this work is to contribute to the development of core skills to the real situation of grades. This contribution is achieved by collecting and selecting electronic resources, which will be classified and structured according to the core skill they contribute to. Other information such as target audience (students, teachers or both) was also collected. Results show a wide quantitative and qualitative divergence among resources corresponding to different skills. This work has a multidisciplinary character, and the objective is not an exhaustive collection of materials, but establishing a more effective organization system of resources for teachers.
\end{abstract}

Keywords: core skills, evaluation, on-line materials, classification, repository

\footnotetext{
Resumen

Una de las principales amenazas detectadas para desarrollar la formación en competencias es la capacidad del profesorado para desarrollarlas en el día a día de sus clases, lo cual puede traducirse en una desmotivación del profesorado ante este reto. En este momento es esencial una importante labor de formación tanto para el profesorado como para el alumno, sobre todo en lo que respecta a cómo trabajar, desarrollar y evaluar las competencias transversales en la realidad de una asignatura determinada. El objetivo general de este trabajo es contribuir a la implementación de las competencias transversales a la realidad de las titulaciones, a través de la recopilación, selección y creación de recursos online y su posterior estructuración y difusión. Así, se ha procedido a recopilar y seleccionar recursos electrónicos ya existentes, clasificándolos según la competencia transversal a la que contribuyan y según su destinatario (profesor o alumno), entre otros. Los resultados muestran una importante disparidad cuantitativa y cualitativa entre recursos correspondientes a distintas competencias. Este
} 
trabajo tiene un carácter marcadamente multidisciplinar, y no tiene como objetivo una recopilación exhaustiva de materiales, sino establecer un sistema de organización de mayor utilidad para el profesorado.

Palabras clave: competencias transversales, evaluación, materiales on-line, clasificación, repositorio.

\section{Introducción}

La educación constituye un pilar esencial de la empleabilidad, entendida como las competencias y cualificaciones transferibles que refuerzan la capacidad de las personas para aprovechar las oportunidades encontrar y conservar un empleo, progresar en la empresa o al cambiar de empleo y adaptarse a la evolución de la tecnología y de las condiciones del mercado de trabajo (OIT, 2004). Proyectos de gran envergadura como REFLEX (Cordis, 2016) o HEGESCO (2016) han demostrado que las empresas no sólo están demandando conocimientos específicos sino también determinadas competencias de carácter más transversal. Sin embargo, se han detectado desajustes entre la formación universitaria y las demandas de los empleadores en cuanto a estas competencias (Martín del Peso et al., 2013, Villarroel y Bruna, 2014). Por todo ello, el desarrollo de las competencias transversales en estudios universitarios ha recibido una creciente atención en los últimos años, en los que numerosas instituciones han establecido iniciativas tratando de incorporar estas competencias como elemento de valor de sus titulaciones (Fallows y Steven, 2000; Alsina, 2011, Villa y Poblete, 2008).

La Universitat Politècnica de València (UPV) también ha establecido en su Plan Estratégico UPV 2015-2020 el objetivo de "ser un referente en docencia y formación de calidad orientada a las necesidades de la Sociedad”. Para alcanzar el objetivo de incorporar las competencias a todos los planes de estudio se han establecido dos planes de acción relacionados con la incorporación de las competencias transversales a los títulos y con la implementación de un sistema de evaluación y acreditación de competencias.

Este proyecto se encuentra en su primer año de implementación y se está enfrentando a los retos esperables de un proyecto de estas características. En concreto, existe un cierto desconcierto en cómo esto puede trasladarse al día a día de profesores y alumnos. En la parte formativa, el profesor se muestra motivado aunque reclama formación al respecto, pero en lo que respecta al sistema de acreditación reclama que se establezcan con claridad y objetividad los criterios de evaluación. Por otra parte, los alumnos son conscientes de la relevancia de la formación en competencias en la medida en que los profesores incluyen en su actividad docente la correspondiente formación y evaluación de competencias transversales.

Por eso mismo es esencial realizar una importante labor de formación tanto a profesores como a alumnos, que en general no están habituados a este tipo de desempeño docente. En definitiva, resulta esencial disponer de los recursos adecuados para trabajar y evaluar las competencias transversales, que resulten de apoyo a los profesores en su tarea docente. Aunque institucionalmente la UPV está desarrollando materiales de apoyo, existe una gran

(cc) EY-NC-ND 2016, Universitat Politècnica de València

Congreso IN-RED (2016) 
cantidad de recursos electrónicos disponibles, tanto en el repositorio institucional de la UPV (riunet) como en otros repositorios. Sin embargo, estos recursos no están catalogados ni organizados en cuanto a su interés de cara a este proyecto de competencias transversales.

Por tanto, este trabajo pretende facilitar la incorporación y evaluación de competencias transversales a la realidad docente de nuestras titulaciones, en el marco del proyecto institucional de la UPV sobre competencias transversales.

\section{Objetivos}

El objetivo de este trabajo establecer una catalogación de los recursos disponibles en Riunet de acuerdo con su aplicabilidad para el aprendizaje y evaluación de competencias transversales que puedan ser aplicados por el profesorado en el día a día.

Para conseguir el objetivo principal se plantean los siguientes objetivos específicos:

1-Identificar y seleccionar materiales on-line existentes sobre competencias transversales.

2-Clasificar los materiales según las distintas competencias a las que hagan referencia y sus posibles destinatarios (profesores o alumnos).

3-Poner a disposición de la comunidad universitaria la selección y clasificación de materiales realizada.

Este trabajo no pretende abarcar la totalidad de competencias a todos sus niveles, sino más bien iniciar un proceso de generación, recopilación, clasificación y divulgación de materiales que constituyan el punto de partida para elaborar un futuro curso de formación on-line en competencias.

\section{Desarrollo de la innovación}

La innovación se está desarrollando durante el curso 2015-2016 y consiste en la búsqueda y catalogación de recursos electrónicos disponibles en https://media.upv.es (principalmente), y así como en otros repositorios relacionados con la Educación Superior. Para organizar la búsqueda, los autores de este trabajo se repartieron las 13 competencias transversales establecidas en la UPV. La búsqueda se realizó según las palabras clave correspondientes a cada competencia.

La clasificación de los recursos se realizó de acuerdo con distintos criterios relacionados con el contenido y utilidad de cada uno. Concretamente se valoraron los siguientes aspectos:

- Título

- Enlace

- Destinatario: Alumno/Profesor/Ambos

- Tipo: Polimedia/Vídeo/Documento/Rúbrica/otros

- Nivel de dominio: 1/2/3/uso general

- Aplicación: Material de apoyo / Ejemplo de aplicación

- Número de visualizaciones (si está disponible)

- Utilidad para otras competencias 
- $\quad$ Ranking cualitativo (1=pobre; 2=regular; 3=bueno; 4=excelente)

- Observaciones

La información recopilada se ha estructurado en una hoja Excel que permite un rápido acceso a la misma. Dado que el proceso de recopilación es continuo, se presentan en este trabajo los resultados hasta el momento del envío del trabajo.

\section{Resultados}

La búsqueda realizada en el repositorio de la UPV ha incluido una buena parte de los recursos electrónicos existentes y ha permitido tener una visión global de cómo las competencias transversales están desarrolladas en los materiales contenidos en el mismo, constatando gran diversidad en cuanto al tipo de materiales y su aplicabilidad. Como resultado se han catalogado 153 recursos relacionados con las distintas competencias. Puede consultarse el documento en https://docs.google.com/spreadsheets/d/1WwlPjyrg_wtwivq-AAS1fZ2_DlGhRwYXBi4Ik5A0H4/edit?usp=sharing.

En la Tabla 1 se muestran el número de recursos en abierto encontrados en el repositorio de la UPV ordenados por competencias transversales. Los materiales no disponibles en abierto no se valoraron por la imposibilidad de seguir la metodología descrita. Dado que el trabajo se encuentra aún en desarrollo, el resultado supone unos resultados preliminares. Sin embargo, estos resultados son ilustrativos de las diferencias de material disponible para cada una de las competencias

De la búsqueda realizada puede destacarse la prolificidad de recursos en algunas áreas, principalmente las relacionadas con el diseño y proyecto, con la innovación y emprendedurismo, con la responsabilidad profesional y la instrumentación. Precisamente en el ámbito instrumental existen multitud de recursos elaborados como materiales de apoyo para utilizar las distintas herramientas de cada titulación. Por el contrario, competencias de carácter más metacognitivo tienen un menor número de recursos (por ejemplo pensamiento crítico, aprendizaje permanente, comprensión e integración...).

Igualmente es destacable la vigencia de gran parte de los materiales encontrados independientemente de su fecha de elaboración, excepto en la competencia instrumental específica, en la que se detectó una acelerada obsolescencia de los materiales de apoyo a herramientas informáticas. Esto en parte explica también la elevada cantidad de materiales producidos, incluso con contenidos duplicados.

También se ha detectado una gran disparidad en el grado de profundidad en que se contribuye al desarrollo de la competencia, desde algunos recursos muy básicos (por ejemplo en recursos sobre temas instrumentales) hasta otros muy elaborados, constituyendo auténticos manuales y organizados en módulos de aprendizaje.

Las competencias con un carácter más cognitivo, es decir, aquellas relacionadas con la comprensión, el análisis, la aplicación, resolución de problemas y el pensamiento crítico son aquellas menos trabajadas expresamente en los recursos electrónicos existentes, encontrándose mayormente algunos ejemplos de aplicación. Probablemente estas competencias son precisamente las que resultan más difíciles en cuanto a la definición de

(cc) EY-NC-ND 2016, Universitat Politècnica de València 
métodos de trabajo y recogida de evidencias, puesto que implican una valoración del proceso cognitivo del propio alumno. Por el contrario, las otras competencias son aparentemente más fácilmente valorables en función de resultados observables.

Tabla 1. Número de recursos electrónicos encontrados en el repositorio de la UPV ordenados por competencias transversales

\begin{tabular}{|l|c|}
\hline Competencia & Número de recursos \\
\hline C1. Comprensión e integración & 3 \\
\hline C2. Aplicación y pensamiento práctico & 0 \\
\hline C3. Análisis y resolución de problemas & 6 \\
\hline C4. Innovación, creatividad y emprendimiento & $21^{*}$ \\
\hline C5. Diseño y proyecto & 5 \\
\hline C6. Trabajo en equipo y liderazgo & $59^{* *}$ \\
\hline C7. Responsabilidad ética, medioambiental y profesional & 7 \\
\hline C8. Comunicación efectiva & 7 \\
\hline C9. Pensamiento crítico & 4 \\
\hline C10. Conocimiento de problemas contemporáneos & 1 \\
\hline C11. Aprendizaje permanente & 9 \\
\hline C12. Planificación y gestión del tiempo & $35^{*}$ \\
\hline C13. Instrumental específica & \\
\hline * Incluye varios recursos dentro de módulos de aprendizaje \\
** Incluye trabajos del repositorio Riunet (https://riunet.upv.es/) \\
\hline
\end{tabular}

Finalmente, como es lógico debido a la finalidad con la que son producidos, cabe destacar que muchos de los recursos encontrados trabajan las competencias de forma colateral y en forma de ejemplos dirigidos a alumnos de titulaciones concretas, por lo que la aplicabilidad general es más bien reducida. Probablemente sería recomendable incluir en la elaboración de los nuevos materiales incluidos en este repositorio un campo descriptivo que indique en su caso la contribución a diferencias competencias transversales, de forma que la búsqueda y catalogación de las mismas sea más eficiente. Esto contribuiría a una búsqueda más ágil por parte de los docentes y alumnos interesados en trabajar las distintas competencias. 


\section{Conclusiones}

De la revisión de recursos electrónicos disponibles en el repositorio de la UPV se ha constatado una gran disparidad en cuanto a su correspondencia con las distintas competencias transversales, existiendo algunas muy representadas mientras que otras apenas se trabajan o se hace de forma colateral y aplicada a ejemplos concretos.

Es fundamental mejorar la producción y catalogación de materiales para contribuir al desarrollo de las competencias transversales.

\section{Referencias}

ALSINA, J. (coord). (2011). Evaluación por competencias en la Universidad: las competencias transversales. Universitat de Barcelona, Institut de Ciències de la Educació. Ediciones Octaedro. 64 p.

CORDIS (2016). Final Report Summary - REFLEX (The flexible professional in the knowledge society new demands on higher education in Europe). Community Research and Development Information Service, European Commission. Available on-line in http://cordis.europa.eu/result/rcn/47889_en.html (accessed 17th May 2016)

FALLOWS, S., STEVEN, C. (2000). Building employability skills into the higher education curriculum: a university-wide initiative. Education + Training 42, 75-82

HEGESCO (2016). Higher Education as a Generator of Strategic Competences. EU Lifelong Learning Programme. Available on-line in http://www.hegesco.org/ (Accessed $17^{\text {th }}$ May 2016).

MARTÍN DEL PESO, M., RABADÁN, A.B., HERNÁNDEZ-MARCH, J. (2013). Desajustes entre formación y empleo en elámbito de las enseñanzas técnicas universitarias: la visión de los empleadores de la Comunidad de Madrid. Revista de Educación, 360.

OIT (2004). Recomendación 195 sobre el desarrollo de los recursos humanos: educación, formación y aprendizaje permanente. Organización Internacional del Trabajo. Adoptado en Ginebra, 92a Reunión del CIT. http://www.ilo.org/ilolex/cgi-lex/convds.pl?R195

VILLA, A., POBLETE, M. (2008). Aprendizaje basado en competencias: una propuesta para la evaluación de las competencias genéricas. Universidad de Deusto, Ed. Mensajero. Bilbao, 333 pág.

VILLARROEL, V., BRUNA, D. (2014). Reflexiones entorno a las competencias genéricas en educación superior: un desafío pendiente. Psicoperspectivas 13, 23-34.

UPV (2014). Plan Estratégico UPV 2015-2020. Universitat Politècnica de València. 43p. A nivel práctico, se han establecido en este proyecto institucional las siguientes competencias transversales: 